\title{
Nontrivial Solutions of a Fully Fourth-Order Periodic Boundary Value Problem
}

\author{
Haitong Li, Minghe Pei, and Libo Wang \\ Department of Mathematics, Beihua University, Jilin City 132013, China \\ Correspondence should be addressed to Minghe Pei; peiminghe@163.com \\ Received 15 May 2014; Accepted 23 July 2014; Published 6 August 2014 \\ Academic Editor: Daqing Jiang
}

Copyright ( 2014 Haitong Li et al. This is an open access article distributed under the Creative Commons Attribution License, which permits unrestricted use, distribution, and reproduction in any medium, provided the original work is properly cited.

We investigate the solvability of a fully fourth-order periodic boundary value problem of the form $x^{(4)}=f\left(t, x, x^{\prime}, x^{\prime \prime}, x^{\prime \prime \prime}\right), x^{(i)}(0)=$ $x^{(i)}(T), i=0,1,2,3$, where $f:[0, T] \times \mathbb{R}^{4} \rightarrow \mathbb{R}$ satisfies Carathéodory conditions. By using the coincidence degree theory, the existence of nontrivial solutions is obtained. Meanwhile, as applications, some examples are given to illustrate our results.

\section{Introduction}

In this paper, we consider a fully nonlinear fourth-order periodic boundary value problem of the form

$$
x^{(4)}=f\left(t, x, x^{\prime}, x^{\prime \prime}, x^{\prime \prime \prime}\right) \text {, }
$$

subject to the boundary conditions

$$
x^{(i)}(0)=x^{(i)}(T), \quad i=0,1,2,3,
$$

where $f:[0, T] \times \mathbb{R}^{4} \rightarrow \mathbb{R}=(-\infty,+\infty)$ satisfies Carathéodory conditions; that is,

(i) for a.e. $t \in[0, T]$, the function $f\left(t, x_{0}, x_{1}, x_{2}, x_{3}\right)$ : $\mathbb{R}^{4} \rightarrow \mathbb{R}$ is continuous;

(ii) for every $\left(x_{0}, x_{1}, x_{2}, x_{3}\right) \in \mathbb{R}^{4}$, the function $f\left(t, x_{0}, x_{1}, x_{2}, x_{3}\right):[0, T] \rightarrow \mathbb{R}$ is measurable;

(iii) for each $\rho>0$, there is a real valued function $h_{\rho}(\cdot) \in$ $L^{1}[0, T]$ such that

$$
\left|f\left(t, x_{0}, x_{1}, x_{2}, x_{3}\right)\right| \leq h_{\rho}(t),
$$

for a.e. $t \in[0, T]$ and $x_{0}^{2}+x_{1}^{2}+x_{2}^{2}+x_{3}^{2}<\rho^{2}$.

It is well known that fourth-order periodic boundary value problems are important research topics which arise in a variety of different areas, such as nonlinear oscillations, fluid mechanical, and nonlinear elastic mechanical phenomena, and thus have been extensively studied; for instance, see [130] and references therein. However, most of the works in the above-mentioned references allow only having $t, x$ or $t, x, x^{\prime \prime}$ in the right-hand side nonlinear function $f$; see $[2-11,13,15-$ $18,20-30]$. The works on the fully nonlinear cases of which $f$ contains explicitly $t$ and every derivative of $x$ up to order three have been quite rarely seen; see $[1,12,14,19]$.

The aim of this paper is to establish the existence of solutions and nontrivial solutions for the fully nonlinear fourth-order PBVP (1), (2). Our main tool is the coincidence degree theory. The paper [31] motivated our study.

\section{Preliminary}

In this section, we present some lemmas which are needed for our main results.

At first, we will briefly recall some notations that are needed for our discussion.

Let $X, Z$ be real Banach spaces. A linear mapping $L$ : $\operatorname{dom} L \subset X \rightarrow Z$ will be called a Fredholm mapping of index zero if the following two conditions hold:

(i) $\operatorname{Im} L$ is a closed subspace of $Z$;

(ii) $\operatorname{dim} \operatorname{Ker} L=\operatorname{codim} \operatorname{Im} L<+\infty$. 
Let $L: \operatorname{dom} L \subset X \rightarrow Z$ be a Fredholm mapping of index zero; then there exist continuous projectors $P: X \rightarrow X$ and $Q: Z \rightarrow Z$ such that

$$
\operatorname{Im} P=\operatorname{Ker} L, \quad \operatorname{Ker} Q=\operatorname{Im} L,
$$

so that

$$
X=\operatorname{Ker} L \oplus \operatorname{Ker} P, \quad Z=\operatorname{Im} L \oplus \operatorname{Im} Q .
$$

It follows that $\left.L\right|_{\operatorname{dom} L \cap \operatorname{Ker} P}: \operatorname{dom} L \cap \operatorname{Ker} P \rightarrow \operatorname{Im} L$ is invertible. We denote the inverse of that map by $K_{P}$. Let $\Omega$ be an open bounded subset of $X$ such that $\operatorname{dom} L \cap \Omega \neq \emptyset$; the map $N: X \rightarrow Z$ will be called $L$-compact on $\bar{\Omega}$, if $Q N: \bar{\Omega} \rightarrow Z$ and $K_{P}(I-Q) N: \bar{\Omega} \rightarrow X$ are compact.

Lemma 1 (see [32]). Let $L: \operatorname{dom} L \rightarrow Z$ be a linear Fredholm mapping of index zero and let $\Omega \subset X$ be an open bounded set. Let $N: \bar{\Omega} \rightarrow Z$ be L-compact on $\bar{\Omega}$ and let $A: X \rightarrow Z$ be L-completely continuous such that

(i) $\operatorname{Ker}(L-A)=\{0\}$;

(ii) for every $(x, \lambda) \in(\operatorname{dom} L \cap \partial \Omega) \times(0,1)$,

$$
L x-(1-\lambda) A x-\lambda N x \neq 0,
$$

and assume that $0 \in \Omega$. Then equation

$$
L x=N x
$$

has at least one solution in $\operatorname{dom} L \cap \bar{\Omega}$.

Lemma 2 (see [33]). Let $L: \operatorname{dom} L \rightarrow Z$ be a linear Fredholm mapping of index zero and let $\Omega \subset X$ be an open bounded set. Let $N: \bar{\Omega} \rightarrow Z$ be L-compact on $\bar{\Omega}$ and the coincidence degree $D[(L, N), \Omega]$ is well defined. If there exists $z \in Z$ with $z \neq 0$ such that

$$
L x-N x \neq \lambda z, \quad \forall(x, \lambda) \in(\operatorname{dom} L \cap \partial \Omega) \times[0,+\infty),
$$

then $D[(L, N), \Omega]=0$.

In the following, we take Banach space $X=C^{3}[0, T]$ with the norm $\|x\|_{C^{3}}=\sum_{i=0}^{3}\left\|x^{(i)}\right\|_{\infty}$, and $Z=L^{1}[0, T]$. Define a linear map $L: \operatorname{dom} L \subset X \rightarrow Z$ by

$$
(L x)(t)=x^{(4)}(t), \quad \forall x \in \operatorname{dom} L,
$$

where $\operatorname{dom} L=\left\{x \in W^{4,1}(0, T): x^{(i)}(0)=x^{(i)}(T), i=\right.$ $0,1,2,3\}$ and $W^{4,1}(0, T)$ is the usual Sobolev space. It is easy to see that $L$ is a Fredholm mapping of index zero. Also define a nonlinear map $N: X \rightarrow Z$ by

$$
(N x)(t)=f\left(t, x, x^{\prime}, x^{\prime \prime}, x^{\prime \prime \prime}\right), \quad \forall x \in X .
$$

Define two projects $P: X \rightarrow X$ and $Q: Z \rightarrow Z$ as follows:

$$
\begin{aligned}
& P x=\frac{1}{T} \int_{0}^{T} x(t) \mathrm{d} t, \quad \forall x \in X ; \\
& Q z=\frac{1}{T} \int_{0}^{T} z(t) \mathrm{d} t, \quad \forall z \in Z .
\end{aligned}
$$

Let $G(t, s)$ be Green function for the homogeneous BVP

$$
\begin{aligned}
x^{(4)}(t) & =0, \quad 0<t<T, \\
\int_{0}^{T} x(t) \mathrm{d} t & =0, \quad x^{(i)}(0)=x^{(i)}(T), \quad i=0,1,2,3 .
\end{aligned}
$$

Then $K_{P}: \operatorname{Im} L \rightarrow \operatorname{dom} L \cap \operatorname{Ker} P$ can be given by

$$
\left(K_{P} z\right)(t)=\int_{0}^{1} G(t, s) z(s) \mathrm{d} s, \quad \forall z \in \operatorname{Im} L .
$$

Hence the $\operatorname{map} K_{P}: \operatorname{Im} L \rightarrow \operatorname{dom} L \cap \operatorname{Ker} P$ is continuous. We note that if $f:[0, T] \times \mathbb{R}^{4} \rightarrow \mathbb{R}$ satisfying Carathéodory conditions, then $N: X \rightarrow Z$ is bounded and continuous by Lebesgue's dominated convergence theorem. Furthermore, $N$ is $L$-compact on every bounded set $\bar{\Omega} \subset X$.

\section{Main Results}

For $h \in L^{1}[0, T]$ and $r \in(0,+\infty)$ we put

$$
\begin{aligned}
& h_{0}=\exp \left(2 \int_{0}^{T} h(t) \mathrm{d} t\right), \quad r_{0}=r+e h_{0} T^{3} ; \\
& \varepsilon \in\left(0, \min \left\{\frac{1}{2 r_{0} T},\left(\frac{2 \pi}{T}\right)^{4}\right\}\right), \quad r_{3}=h_{0} \exp \left(2 \varepsilon r_{0} T\right) ; \\
& r_{2}=r_{3} T, \quad r_{1}=r_{2} T .
\end{aligned}
$$
lemmas.

In order to introduce our main theorem, we need some

Lemma 3. Let $h \in L^{1}[0, T]$ be a nonnegative function and $r \in(0,+\infty)$. Let $r_{0}, r_{1}, r_{2}, r_{3}$, and $\varepsilon$ fulfil (14). Then for any $x \in \operatorname{dom} L$, the inequalities

$$
\begin{array}{r}
|x(t)| \leq r_{0}, \quad\left|x^{\prime}(t)\right| \leq r_{1}, \quad\left|x^{\prime \prime}(t)\right| \leq r_{2}, \\
\forall t \in[0, T], \\
x^{(4)}(t) \operatorname{sign} x^{\prime \prime \prime}(t) \leq h(t)\left|x^{\prime \prime \prime}(t)\right|+\varepsilon|x(t)| \\
\text { for a.e. } t \in\left\{t \in[0, T]:\left|x^{\prime \prime \prime}(t)\right| \geq 1\right\}
\end{array}
$$

imply

$$
\left|x^{\prime \prime \prime}(t)\right|<r_{3}, \quad \forall t \in[0, T] .
$$

Proof. Since $x \in \operatorname{dom} L$, there exists $t_{0} \in(0, T)$ such that

$$
x^{\prime \prime \prime}\left(t_{0}\right)=0
$$

We will show that

$$
\left|x^{\prime \prime \prime}(t)\right|<\sqrt{r_{3}}, \quad \forall t \in\left[t_{0}, T\right] .
$$

By contradiction, assume that there exists $t_{1} \in\left(t_{0}, T\right]$ such that

$$
\left|x^{\prime \prime \prime}\left(t_{1}\right)\right| \geq \sqrt{r_{3}} .
$$


Then there exists $t_{2} \in\left(t_{0}, t_{1}\right)$ such that

$$
\left|x^{\prime \prime \prime}\left(t_{2}\right)\right|=1, \quad\left|x^{\prime \prime \prime}(t)\right| \geq 1, \quad \forall t \in\left[t_{2}, t_{1}\right] .
$$

There are two cases to consider.

Case 1. Consider $x^{\prime \prime \prime}(t) \geq 1$ on $\left[t_{2}, t_{1}\right]$. In this case, integrating (16) from $t_{2}$ to $t_{1}$ and using (15) and (21) we infer that

$$
\int_{t_{2}}^{t_{1}} \frac{x^{(4)}(t)}{x^{\prime \prime \prime}(t)} \mathrm{d} t \leq \int_{t_{2}}^{t_{1}}\left(h(t)+\varepsilon r_{0}\right) \mathrm{d} t<\int_{0}^{T} h(t) \mathrm{d} t+\varepsilon r_{0} T .
$$

Thus $x^{\prime \prime \prime}\left(t_{1}\right)<\sqrt{r_{3}}$, which contradicts (20).

Case 2. Consider $x^{\prime \prime \prime}(t) \leq-1$ on $\left[t_{2}, t_{1}\right]$. Similar to Case 1 , we have

$$
\int_{t_{2}}^{t_{1}} \frac{-x^{(4)}(t)}{-x^{\prime \prime \prime}(t)} \mathrm{d} t \leq \int_{t_{2}}^{t_{1}}\left(h(t)+\varepsilon r_{0}\right) \mathrm{d} t<\int_{0}^{T} h(t) \mathrm{d} t+\varepsilon r_{0} T .
$$

Thus, $x^{\prime \prime \prime}\left(t_{1}\right)>-\sqrt{r_{3}}$, which contradicts (20). Therefore (19) is true. Furthermore, from the fact $x^{\prime \prime \prime}(0)=x^{\prime \prime \prime}(T)$ it follows that $\left|x^{\prime \prime \prime}(0)\right|<\sqrt{r_{3}}$.

Finally, we show that

$$
\left|x^{\prime \prime \prime}(t)\right|<r_{3}, \quad \forall t \in\left[0, t_{0}\right] .
$$

Suppose on the contrary that there exists $t_{3} \in\left(0, t_{0}\right)$ satisfying

$$
\left|x^{\prime \prime \prime}\left(t_{3}\right)\right| \geq r_{3} .
$$

Then there exists $t_{4} \in\left(0, t_{3}\right)$ such that

$$
\left|x^{\prime \prime \prime}\left(t_{4}\right)\right|=\sqrt{r_{3}}, \quad\left|x^{\prime \prime \prime}(t)\right| \geq \sqrt{r_{3}}, \quad \forall t \in\left[t_{4}, t_{3}\right] .
$$

There are two cases to consider.

Case $1^{\prime}$. Consider $x^{\prime \prime \prime}(t) \geq \sqrt{r_{3}}$ on $\left[t_{4}, t_{3}\right]$. Similar to Case 1 , we have $x^{\prime \prime \prime}\left(t_{3}\right)<r_{3}$, which contradicts (25).

Case $2^{\prime}$. Consider $x^{\prime \prime \prime}(t) \leq-\sqrt{r_{3}}$ on $\left[t_{4}, t_{3}\right]$. Similar to Case 2 , one has $x^{\prime \prime \prime}\left(t_{3}\right)>-r_{3}$, which also contradicts (25). Hence (24) is true.

In summary, from (19) and (24) it follows that estimate (17) holds. This completes the proof of the lemma.

Lemma 4. Let $r \in(0,+\infty)$ and let $h \in L^{1}[0, T]$ be a nonnegative function. Let $r_{0}, r_{1}, r_{2}, r_{3}$, and $\varepsilon$ fulfil (14). Then for any function $x \in \operatorname{dom} L$ the inequalities

$$
\left|x^{\prime \prime \prime}(t)\right|<r_{3}, \quad \forall t \in[0, T],
$$

$$
\mu x^{(4)}(t) \operatorname{sign} x(t)>0 \quad \text { for a.e. } t \in\{t \in[0, T]:|x(t)| \geq r\}
$$

imply

$$
\begin{array}{r}
|x(t)|<r_{0}, \quad\left|x^{\prime}(t)\right|<r_{1}, \quad\left|x^{\prime \prime}(t)\right|<r_{2}, \\
\forall t \in[0, T] .
\end{array}
$$

Proof. For every $x \in \operatorname{dom} L$, from (28), there exist $t_{0}, t_{1}, t_{2} \in$ $[0, T]$ such that

$$
\left|x\left(t_{0}\right)\right|<r, \quad x^{\prime}\left(t_{1}\right)=0, \quad x^{\prime \prime}\left(t_{2}\right)=0 .
$$

Integrating (27) by (14) and (30) we get

$$
\begin{aligned}
& \left|x^{\prime \prime}(t)\right|<r_{3} T=r_{2}, \quad\left|x^{\prime}(t)\right|<r_{2} T=r_{1} \quad \text { on }[0, T], \\
& |x(t)|<r+r_{2} T^{2}<r_{0} \quad \text { on }[0, T] .
\end{aligned}
$$

This completes the proof of the lemma.

Now, we apply Lemma 1 to establish the existence results of solutions for the fourth-order PBVP (1), (2).

Theorem 5. Assume that there exist $\mu \in\{-1,1\}, r \in(0,+\infty)$, and a nonnegative function $h \in L^{1}[0, T]$. Suppose further that

$\left(\mathrm{H}_{0}\right) f:[0, T] \times \mathbb{R}^{4} \rightarrow \mathbb{R}$ satisfies the Carathéodory conditions;

$\left(\mathrm{H}_{1}\right)$ if $\left|x_{0}\right| \geq r,\left|x_{1}\right| \leq r_{1},\left|x_{2}\right| \leq r_{2},\left|x_{3}\right| \leq r_{3}$, then

$\mu f\left(t, x_{0}, x_{1}, x_{2}, x_{3}\right) \operatorname{sign} x_{0} \geq 0 \quad$ for a.e. $t \in[0, T] ;$

$\left(\mathrm{H}_{2}\right)$ if $\left|x_{0}\right| \leq r_{0},\left|x_{1}\right| \leq r_{1},\left|x_{2}\right| \leq r_{2},\left|x_{3}\right| \geq 1$, then

$f\left(t, x_{0}, x_{1}, x_{2}, x_{3}\right) \operatorname{sign} x_{3} \leq h(t)\left|x_{3}\right| \quad$ for a.e. $t \in[0, T]$,

where $r_{0}, r_{1}, r_{2}, r_{3}$ fulfil (14). Then PBVP (1), (2) has at least one solution $x$ such that

$$
\left|x^{(i)}(t)\right| \leq r_{i}, \quad i=0,1,2,3, \forall t \in[0, T] .
$$

Proof. Let

$$
\Omega=\left\{x \in X:\left|x^{(i)}(t)\right|<r_{i}, i=0,1,2,3, t \in[0, T]\right\} .
$$

Then $x \in \partial \Omega$ iff there exist some $j \in\{0,1,2,3\}$ such that

$$
\begin{array}{r}
\max _{0 \leq t \leq T}\left|x^{(j)}(t)\right|=r_{j}, \quad\left|x^{(i)}(t)\right| \leq r_{i}, \quad i=0,1,2,3, \\
t \in[0, T] .
\end{array}
$$

Now, we show that

$$
\operatorname{Ker}(L-A)=\{0\},
$$

where $A: X \rightarrow Z, x \mapsto \mu \varepsilon x$. To do this, we assume that $x(t)$ is the solution of the following periodic boundary value problem:

$$
\begin{aligned}
& x^{(4)}(t)=\mu \varepsilon x(t), \quad \text { a.e. } t \in(0, T), \\
& x^{(i)}(0)=x^{(i)}(T), \quad i=0,1,2,3 .
\end{aligned}
$$

Integrating the equation as above on $[0, T]$, we obtain

$$
\int_{0}^{T} x(t) \mathrm{d} t=0
$$


Thus, by Wirtinger inequality,

$$
\left\|x^{(i)}\right\|_{2} \leq \frac{T}{2 \pi}\left\|x^{(i+1)}\right\|_{2}, \quad i=0,1,2,3 .
$$

Hence from (38) it follows that

$$
\|x\|_{2} \leq\left(\frac{T}{2 \pi}\right)^{4}\left\|x^{(4)}\right\|_{2} \leq \varepsilon\left(\frac{T}{2 \pi}\right)^{4}\|x\|_{2} .
$$

If $\|x\|_{2}>0$, then, from $\varepsilon<(2 \pi / T)^{4}$, the following contradiction holds:

$$
\|x\|_{2}<\|x\|_{2} .
$$

Therefore, $\operatorname{Ker}(L-A)=\{0\}$.

Finally, we show that, for every $(x, \lambda) \in(\operatorname{dom} L \cap \partial \Omega) \times$ $(0,1)$,

$$
L x-(1-\lambda) A x-\lambda N x \neq 0 .
$$

To do this, let $\lambda \in(0,1)$ and let $x_{\lambda} \in \bar{\Omega}$ be a solution of the following PBVP:

$$
\begin{aligned}
x^{(4)} & =\lambda f\left(t, x, x^{\prime}, x^{\prime \prime}, x^{\prime \prime \prime}\right)+(1-\lambda) \mu \varepsilon x, \\
x^{(i)}(0) & =x^{(i)}(T), \quad i=0,1,2,3 .
\end{aligned}
$$

Then $x_{\lambda} \notin \partial \Omega$. In fact, let

$$
\begin{array}{r}
\left|x_{\lambda}(t)\right| \leq r_{0}, \quad\left|x_{\lambda}^{\prime}(t)\right| \leq r_{1}, \quad\left|x_{\lambda}^{\prime \prime}(t)\right| \leq r_{2}, \\
\forall t \in[0, T] .
\end{array}
$$

Then, by (33),

$$
\begin{aligned}
x_{\lambda}^{(4)} \operatorname{sign} x_{\lambda}^{\prime \prime \prime}= & \lambda f \operatorname{sign} x_{\lambda}^{\prime \prime \prime} \\
& +(1-\lambda) \mu \varepsilon x_{\lambda} \operatorname{sign} x_{\lambda}^{\prime \prime \prime} \leq h(t)\left|x_{\lambda}^{\prime \prime \prime}\right|+\varepsilon\left|x_{\lambda}\right|,
\end{aligned}
$$

for a.e. $t \in\left\{t \in[0, T]:\left|x_{\lambda}^{\prime \prime \prime}(t)\right| \geq 1\right\}$. Applying Lemma 3, we obtain

$$
\left|x_{\lambda}^{\prime \prime \prime}(t)\right|<r_{3}, \quad \forall t \in[0, T] .
$$

Thus according to (32), we have

$$
\mu x_{\lambda}^{(4)} \operatorname{sign} x_{\lambda}=\lambda \mu f \operatorname{sign} x_{\lambda}+\mu^{2}(1-\lambda) \varepsilon\left|x_{\lambda}\right|>0,
$$

for a.e. $t \in\left\{t \in[0, T]:\left|x_{\lambda}(t)\right| \geq r\right\}$. It follows from Lemma 4 that

$$
\begin{array}{r}
\left|x_{\lambda}(t)\right|<r_{0}, \quad\left|x_{\lambda}^{\prime}(t)\right|<r_{1}, \quad\left|x_{\lambda}^{\prime \prime}(t)\right|<r_{2}, \\
\forall t \in[0, T] .
\end{array}
$$

Thus $x_{\lambda} \notin \partial \Omega$. This implies that condition (ii) of Lemma 1 is valid.

In summary, all conditions of Lemma 1 are satisfied. Therefore the conclusion of Theorem 5 holds. This completes the proof of the theorem.
Next, we establish the existence result of nontrivial solutions for the fourth-order PBVP (1), (2) by means of Lemma 2.

Theorem 6. Assume that all conditions in Theorem 5 hold with the exception of $\left(H_{1}\right)$, which is replaced by the following:

$$
\begin{aligned}
& \left(\mathrm{H}_{1}^{\prime}\right) \text { there exists a constant } r^{*} \in(0, r) \text { such that if } x_{0}>-r^{*}, \\
& \quad\left|x_{1}\right| \leq r_{1},\left|x_{2}\right| \leq r_{2},\left|x_{3}\right| \leq r_{3} \text {, then } \\
& \mu f\left(t, x_{0}, x_{1}, x_{2}, x_{3}\right) \geq 0 \quad \text { for a.e. } t \in[0, T], \\
& \quad \text { and if } x_{0}<-r,\left|x_{1}\right| \leq r_{1},\left|x_{2}\right| \leq r_{2},\left|x_{3}\right| \leq r_{3} \text {, then } \\
& \mu f\left(t, x_{0}, x_{1}, x_{2}, x_{3}\right) \leq 0 \quad \text { for a.e. } t \in[0, T] .
\end{aligned}
$$

Then PBVP (1), (2) has at least one nontrivial solution $x$ satisfying (34).

Proof. From the proof of Theorem 5 and Lemma 1, it follows that $L x=N x$ has a solution in

$$
\Omega=\left\{x \in X:\left|x^{(i)}(t)\right|<r_{i}, i=0,1,2,3, t \in[0, T]\right\},
$$

and $|D[(L, N), \Omega]|=1$. Without loss of generality, we assume that $\mu=1$ and $B_{r^{*}}=\left\{x:\|x\|_{C^{3}}<r^{*}\right\} \subset \Omega$. We also assume that $L x \neq N x$ for all $x \in \partial B_{r^{*}}$.

Now we assert that

$$
D\left[(L, N), B_{r^{*}}\right]=0 .
$$

In fact, suppose that there exist $x_{0} \in \partial B_{r^{*}}$ and $\lambda_{0}>0$ such that

$$
L x_{0}-N x_{0}=\lambda_{0}
$$

Applying $Q$ to both sides of above equality, it follows that

$$
-\mathrm{QN} x_{0}=\lambda_{0} \mathrm{Q}(1)
$$

that is,

$$
-\frac{1}{T} \int_{0}^{T} f\left(t, x_{0}(t), x_{0}^{\prime}(t), x_{0}^{\prime \prime}(t), x_{0}^{\prime \prime \prime}(t)\right) \mathrm{d} t=\lambda_{0} .
$$

Notice that $\left\|x_{0}\right\|_{C^{3}}=r^{*}$ and $B_{r^{*}} \subset \Omega$; we have

$$
\begin{array}{rr}
x_{0}(t) \geq-\left\|x_{0}\right\|_{\infty} \geq-\left\|x_{0}\right\|_{C^{3}}=-r^{*}, & \forall t \in[0, T], \\
\left|x_{0}^{\prime}(t)\right| \leq r_{1}, \quad\left|x_{0}^{\prime \prime}(t)\right| \leq r_{2}, \quad & \left|x_{0}^{\prime \prime \prime}(t)\right| \leq r_{3}, \\
& \forall t \in[0, T] .
\end{array}
$$

Consequently, from assumption $\left(\mathrm{H}_{1}^{\prime}\right)$ one has

$$
f\left(t, x_{0}(t), x_{0}^{\prime}(t), x_{0}^{\prime \prime}(t), x_{0}^{\prime \prime \prime}(t)\right) \geq 0 \quad \text { for a.e. } t \in[0, T] \text {. }
$$

This together with (56) it follows that

$$
\lambda_{0} \leq 0
$$


which is a contradiction. This implies that

$$
L x-N x \neq \lambda, \quad \forall x \in \partial B_{r^{*}}, \forall \lambda \geq 0 .
$$

Thus from Lemma 2 it follows that

$$
D\left[(L, N), B_{r^{*}}\right]=0 .
$$

Hence

$$
D\left[(L, N), \Omega \backslash \bar{B}_{r^{*}}\right]=D[(L, N), \Omega]-D\left[(L, N), B_{r^{*}}\right] \neq 0 .
$$

Therefore $L x=N x$ has a solution in $\Omega \backslash \bar{B}_{r^{*}}$; that is, PBVP (1), (2) has at least one nontrivial solution. This completes the proof of the theorem.

Finally, we give some examples to illustrate our results.

Example 7. Consider the fourth-order periodic boundary value problem

$$
\begin{aligned}
& x^{(4)}(t)=\lambda h(t)(x(t)+1) e^{x(t) x^{\prime}(t)}\left(x^{\prime \prime}(t)\right)^{2}\left(x^{\prime \prime \prime}(t)+c\right), \\
& \text { a.e. } t \in[0, T], \\
& x^{(i)}(0)=x^{(i)}(T), \quad i=0,1,2,3,
\end{aligned}
$$

where $\lambda$ is a parameter, $h \in L^{1}[0, T]$ is nonnegative, and

$$
c \in\left(\exp \left(2 \int_{0}^{T} h(t) \mathrm{d} t+1\right),+\infty\right)
$$

is a constant.

Let

$$
f\left(t, x_{0}, x_{1}, x_{2}, x_{3}\right)=\lambda h(t)\left(x_{0}+1\right) e^{x_{0} x_{1}} x_{2}^{2}\left(x_{3}+c\right) .
$$

Then $f:[0, T] \times \mathbb{R}^{4} \rightarrow \mathbb{R}$ satisfies Carathéodory conditions. Taking any $r \in(1,+\infty)$, then $r_{0}, r_{1}, r_{2}$, and $r_{3}$ are well defined by (14).

Now, we assert that all conditions of Theorem 5 are satisfied when

$$
\lambda \in\left[-\frac{1}{\left(r_{0}+1\right) e^{r_{0} r_{1}} r_{2}^{2}\left(r_{3}+c\right)}, \frac{1}{\left(r_{0}+1\right) e^{r_{0} r_{1}} r_{2}^{2}\left(r_{3}+c\right)}\right] .
$$

In fact, without loss of generality, we can assume $\lambda \in\left[-1 /\left(r_{0}+\right.\right.$ 1) $\left.e^{r_{0} r_{1}} r_{2}^{2}\left(r_{3}+c\right), 0\right]$. In this case, we choose $\mu=-1$. It is easy to see that, for every $\left(x_{0}, x_{1}, x_{2}, x_{3}\right) \in[r,+\infty) \times \mathbb{R}^{2} \times\left[-r_{3}, r_{3}\right]$,

$$
\mu f\left(t, x_{0}, x_{1}, x_{2}, x_{3}\right) \operatorname{sign} x_{0} \geq 0, \quad \text { a.e. } t \in[0, T],
$$

and, for every $\left(x_{0}, x_{1}, x_{2}, x_{3}\right) \in(-\infty,-r] \times \mathbb{R}^{2} \times\left[-r_{3}, r_{3}\right]$,

$$
\mu f\left(t, x_{0}, x_{1}, x_{2}, x_{3}\right) \operatorname{sign} x_{0} \geq 0, \quad \text { a.e. } t \in[0, T] .
$$

Hence condition $\left(\mathrm{H}_{1}\right)$ of Theorem 5 is satisfied. In addition, for $\left|x_{0}\right| \leq r_{0},\left|x_{1}\right| \leq r_{1},\left|x_{2}\right| \leq r_{2},\left|x_{3}\right| \geq 1$, we have

$$
\begin{aligned}
f( & \left.t, x_{0}, x_{1}, x_{2}, x_{3}\right) \operatorname{sign} x_{3} \\
& \leq|\lambda| h(t)\left(\left|x_{0}\right|+1\right) e^{\left|x_{0} x_{1}\right|} x_{2}^{2}\left(\left|x_{3}\right|+c\right) \\
& \leq h(t) \frac{\left|x_{3}\right|+c}{r_{3}+c} \\
& \leq h(t)\left|x_{3}\right|, \quad \text { a.e. } t \in[0, T] .
\end{aligned}
$$

Therefore, condition $\left(\mathrm{H}_{2}\right)$ of Theorem 5 is also satisfied. Hence, from Theorem 5, the fourth-order PBVP (63) has at least a solution $x=x(t)$, provided

$$
\lambda \in\left[-\frac{1}{\left(r_{0}+1\right) e^{r_{0} r_{1}} r_{2}^{2}\left(r_{3}+c\right)}, \frac{1}{\left(r_{0}+1\right) e^{r_{0} r_{1}} r_{2}^{2}\left(r_{3}+c\right)}\right] .
$$

Example 8. Consider the fourth-order periodic boundary value problem

$$
\begin{aligned}
& x^{(4)}(t)=\frac{\lambda h(t)(x(t)+1)^{k}\left|x^{\prime \prime \prime}(t)\right|}{1+\left(x^{\prime}(t)\right)^{n}+\left(x^{\prime \prime}(t)\right)^{n}}, \quad \text { a.e. } t \in[0, T], \\
& x^{(i)}(0)=x^{(i)}(T), \quad i=0,1,2,3,
\end{aligned}
$$

where $\lambda$ is a parameter, $k, n \in \mathbb{N}=\{1,2, \ldots\}, k$ is odd, $n$ is even, and $h \in L^{1}[0, T]$ is a nonnegative function.

Let

$$
f\left(t, x_{0}, x_{1}, x_{2}, x_{3}\right)=\frac{\lambda h(t)\left(x_{0}+1\right)^{k}\left|x_{3}\right|}{1+x_{1}^{n}+x_{2}^{n}} .
$$

Then $f:[0, T] \times \mathbb{R}^{4} \rightarrow \mathbb{R}$ satisfies Carathéodory conditions. We choose $r \in(1,+\infty)$; then $r_{0}$ is well defined by (14).

Now, we assert that $f$ satisfies all conditions of Theorem 6 when

$$
\lambda \in\left[-\left(r_{0}+1\right)^{-k},\left(r_{0}+1\right)^{-k}\right] .
$$

In fact, without loss of generality, we can assume that $\lambda \epsilon$ $\left[0,\left(r_{0}+1\right)^{-k}\right]$. Choose $\mu=1$ and $r^{*}=1$. Then it is easy to see that, for every $\left(x_{0}, x_{1}, x_{2}, x_{3}\right) \in\left[-r^{*},+\infty\right) \times \mathbb{R}^{3}$,

$$
\mu f\left(t, x_{0}, x_{1}, x_{2}, x_{3}\right) \geq 0, \quad \text { a.e. } t \in[0, T],
$$

and, for every $\left(x_{0}, x_{1}, x_{2}, x_{3}\right) \in(-\infty,-r] \times \mathbb{R}^{3}$,

$$
\mu f\left(t, x_{0}, x_{1}, x_{2}, x_{3}\right) \leq 0 \quad \text { a.e. } t \in[0, T] .
$$

On the other hand, for every $\left(x_{0}, x_{1}, x_{2}, x_{3}\right) \in\left[-r_{0}, r_{0}\right] \times \mathbb{R}^{3}$,

$$
\begin{aligned}
& f\left(t, x_{0}, x_{1}, x_{2}, x_{3}\right) \operatorname{sign} x_{3} \\
& \quad \leq \frac{\lambda\left|x_{0}+1\right|^{k}}{1+x_{1}^{n}+x_{2}^{n}} h(t)\left|x_{3}\right| \\
& \quad \leq \frac{1}{1+x_{1}^{n}+x_{2}^{n}} h(t)\left|x_{3}\right| \\
& \quad \leq h(t)\left|x_{3}\right|, \quad \text { a.e. } t \in[0, T] .
\end{aligned}
$$


In summary, all conditions of Theorem 6 are satisfied. Therefore, from Theorem 6, the fourth-order PBVP (71) has at least one nontrivial solution $x=x(t)$, provided $\lambda \in\left[-\left(r_{0}+\right.\right.$ $\left.1)^{-k},\left(r_{0}+1\right)^{-k}\right]$.

\section{Conflict of Interests}

The authors declare that there is no conflict of interests regarding the publication of this paper.

\section{Acknowledgment}

This work was supported by the National Natural Science Foundation of China (11201008).

\section{References}

[1] P. Amster and M. C. Mariani, "Oscillating solutions of a nonlinear fourth order ordinary differential equation," Journal of Mathematical Analysis and Applications, vol. 325, no. 2, pp. 1133-1141, 2007.

[2] Z. Bai, "Iterative solutions for some fourth-order periodic boundary value problems," Taiwanese Journal of Mathematics, vol. 12, no. 7, pp. 1681-1690, 2008.

[3] C. Bereanu, "Periodic solutions of some fourth-order nonlinear differential equations," Nonlinear Analysis: Theory, Methods and Applications, vol. 71, no. 1-2, pp. 53-57, 2009.

[4] A. Cabada, "The method of lower and upper solutions for second, third, fourth, and higher order boundary value problems," Journal of Mathematical Analysis and Applications, vol. 185, no. 2, pp. 302-320, 1994.

[5] A. Cabada and S. Lois, "Maximum principles for fourth and sixth order periodic boundary value problems," Nonlinear Analysis: Theory, Methods and Applications, vol. 29, no. 10, pp. 1161-1171, 1997.

[6] A. Cabada and J. J. Nieto, "Quasilinearization and rate of convergence for higher-order nonlinear periodic boundary-value problems," Journal of Optimization Theory and Applications, vol. 108, no. 1, pp. 97-107, 2001.

[7] P. C. Carrião, L. F. O. Faria, and O. H. Miyagaki, "Periodic solutions for extended Fisher-Kolmogorov and Swift-Hohenberg equations by truncature techniques," Nonlinear Analysis: Theory, Methods \& Applications, vol. 67, no. 11, pp. 3076-3083, 2007.

[8] J. Chen and D. O’Regan, "On periodic solutions for even order differential equations," Nonlinear Analysis: Theory, Methods \& Applications, vol. 69, no. 4, pp. 1138-1144, 2008.

[9] F. Cong, "Periodic solutions for $2 k$ th order ordinary differential equations with nonresonance," Nonlinear Analysis: Theory, Methods and Applications, vol. 32, no. 6, pp. 787-793, 1998.

[10] M. Conti, S. Terracini, and G. Verzini, "Infinitely many solutions to fourth order superlinear periodic problems," Transactions of the American Mathematical Society, vol. 356, no. 8, pp. 32833300, 2004.

[11] Q. Fan, W. Wang, and J. Zhou, "Periodic solutions of some fourth-order nonlinear differential equations," Journal of Computational and Applied Mathematics, vol. 233, no. 2, pp. 121-126, 2009.

[12] J. Fialho and F. Minhós, "On higher order fully periodic boundary value problems," Journal of Mathematical Analysis and Applications, vol. 395, no. 2, pp. 616-625, 2012.
[13] M. R. Grossinho, L. Sanchez, and S. A. Tersian, "On the solvability of a boundary value problem for a fourth-order ordinary differential equation," Applied Mathematics Letters, vol. 18, no. 4, pp. 439-444, 2005.

[14] C. P. Gupta, "Solvability of a fourth-order boundary value problem with periodic boundary conditions II," International Journal of Mathematics and Mathematical Sciences, vol. 14, pp. 127-138, 1991.

[15] D. Jiang, W. Gao, and A. Wan, "A monotone method for constructing extremal solutions to fourth-order periodic boundary value problems," Applied Mathematics and Computation, vol. 132, no. 2-3, pp. 411-421, 2002.

[16] Y. Li, "Positive solutions of fourth-order periodic boundary value problems," Nonlinear Analysis. Theory, Methods \& Applications. An International Multidisciplinary Journal. Series A: Theory and Methods, vol. 54, no. 6, pp. 1069-1078, 2003.

[17] Y. Li, "On the existence and uniqueness for higher order periodic boundary value problems," Nonlinear Analysis: Theory, Methods and Applications, vol. 70, no. 2, pp. 711-718, 2009.

[18] Y. Liu, "Solvability of periodic boundary value problems for $n$ thorder ordinary differential equations," Computers \& Mathematics with Applications, vol. 52, no. 6-7, pp. 1165-1182, 2006.

[19] Z. Liu, "Periodic solutions for nonlinear $n$th order ordinary differential equations," Journal of Mathematical Analysis and Applications, vol. 204, no. 1, pp. 46-64, 1996.

[20] S. Lu and S. Jin, "Existence of periodic solutions for a fourthorder $p$-Laplacian equation with a deviating argument," Journal of Computational and Applied Mathematics, vol. 230, no. 2, pp. 513-520, 2009.

[21] R. Y. Ma, "Solvability of a class of fourth-order periodic boundary value problems," Acta Mathematica Scientia. Series A, vol. 15, no. 3, pp. 315-318, 1995.

[22] J. Mawhin and F. Zanolin, "A continuation approach to fourth order superlinear periodic boundary value problems," Topological Methods in Nonlinear Analysis, vol. 2, no. 1, pp. 55-74, 1993.

[23] F. I. Njoku and P. Omari, "Singularly perturbed higher order periodic boundary value problems," Journal of Mathematical Analysis and Applications, vol. 289, no. 2, pp. 639-649, 2004.

[24] L. A. Peletier and W. C. Troy, "Spatial patterns described by the extended Fisher-Kolmogorov equation: periodic solutions," SIAM Journal on Mathematical Analysis, vol. 28, no. 6, pp. 13171353, 1997.

[25] V. Šeda, J. J. Nieto, and M. Gera, "Periodic boundary value problems for nonlinear higher order ordinary differential equations," Applied Mathematics and Computation, vol. 48, no. 1, pp. 71-82, 1992.

[26] S. Tersian and J. Chaparova, "Periodic and homoclinic solutions of extended Fisher-Kolmogorov equations," Journal of Mathematical Analysis and Applications, vol. 260, no. 2, pp. 490-506, 2001.

[27] Z. Wang, L. Qian, and S. Lu, "On the existence of periodic solutions to a fourth-order $p$-Laplacian differential equation with a deviating argument," Nonlinear Analysis: Real World Applications, vol. 11, no. 3, pp. 1660-1669, 2010.

[28] J. R. Ward Jr., "Asymptotic conditions for periodic solutions of ordinary differential equations," Proceedings of the American Mathematical Society, vol. 81, no. 3, pp. 415-420, 1981.

[29] Q. Yao, "Existence, multiplicity and infinite solvability of positive solutions to a nonlinear fourth-order periodic boundary value problem," Nonlinear Analysis: Theory, Methods and Applications, vol. 63, no. 2, pp. 237-246, 2005. 
[30] C. Zhao, W. Chen, and J. Zhou, "Periodic solutions for a class of fourth-order nonlinear differential equations," Nonlinear Analysis: Theory, Methods \& Applications, vol. 72, no. 3-4, pp. 1221-1226, 2010.

[31] I. Rachůnková, "Periodic boundary value problems for thirdorder differential equations," Mathematica Slovaca, vol. 41, no. 3, pp. 241-248, 1991.

[32] J. Mawhin, Topological Degree Methods in Nonlinear Boundary Value Problems, vol. 40 of NSF-CBMS Regional Conference Series in Mathematics, American Mathematical Society, Providence, RI, USA, 1979.

[33] F. B. Zhang, "Lack of direction property of the coincidence degree and applications," Journal of Mathematical Research and Exposition, vol. 19, no. 4, pp. 693-698, 1999 (Chinese). 


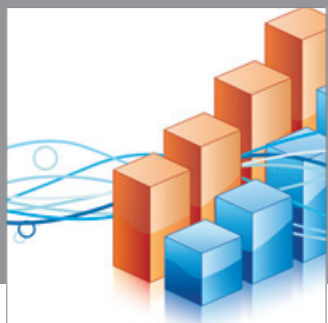

Advances in

Operations Research

mansans

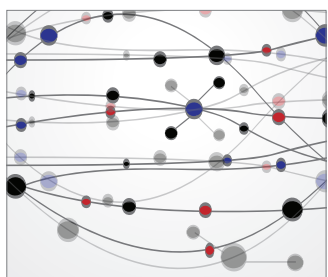

The Scientific World Journal
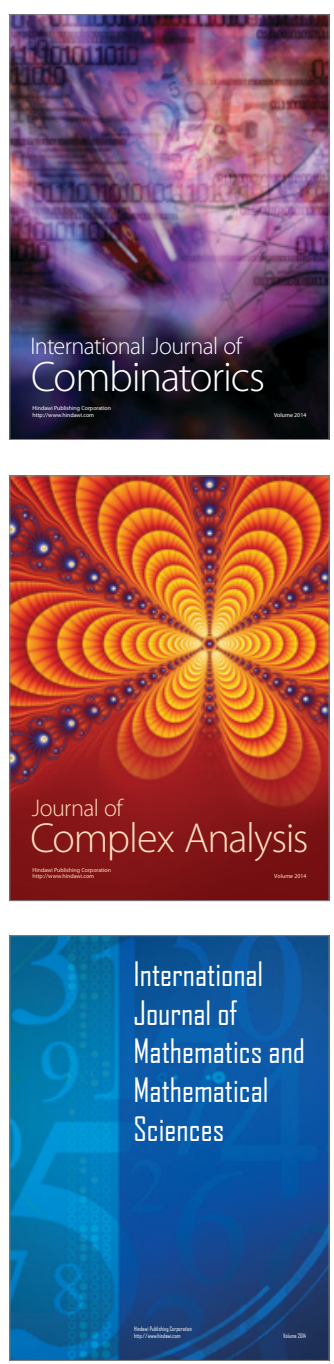
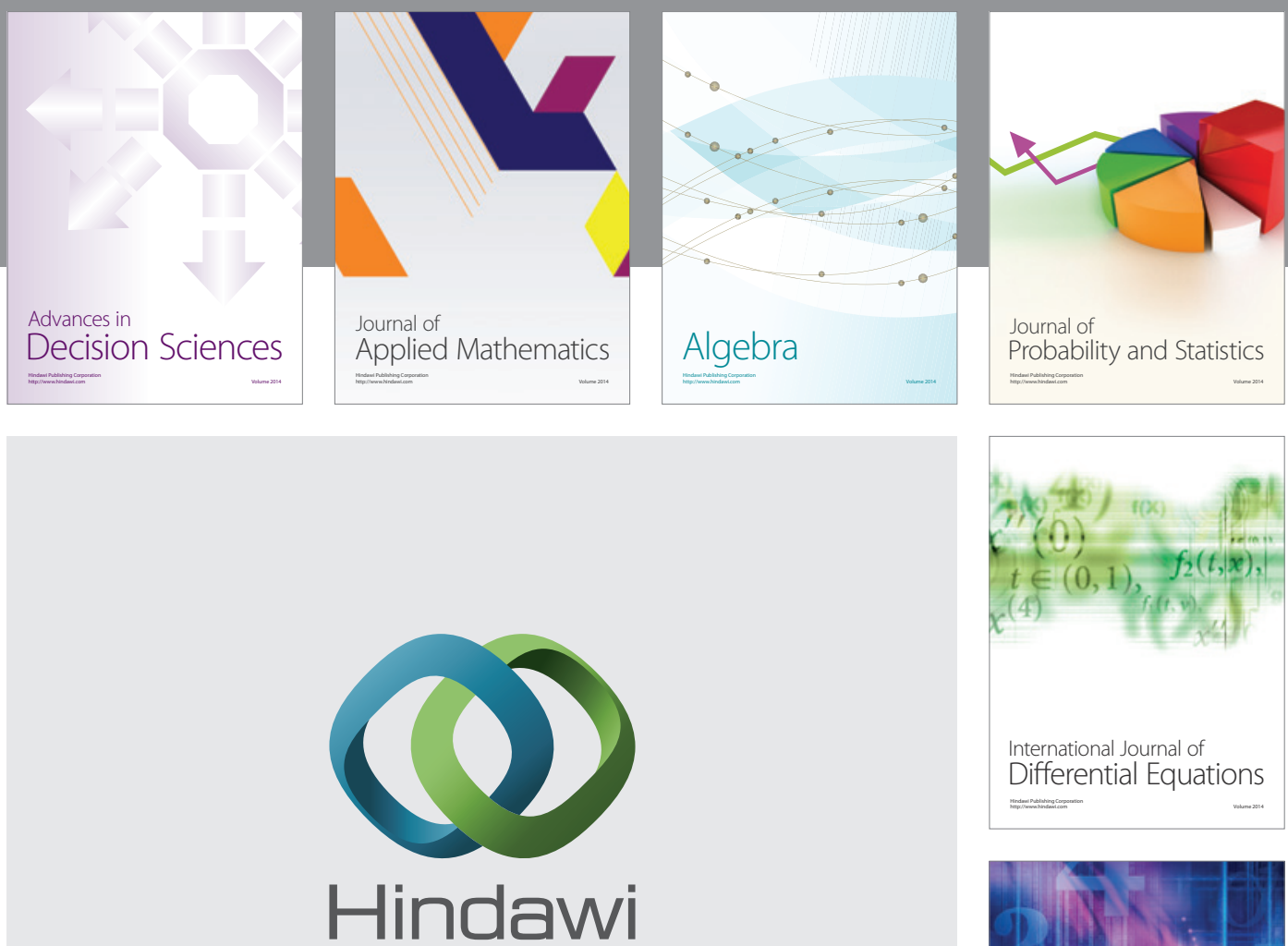

Submit your manuscripts at http://www.hindawi.com
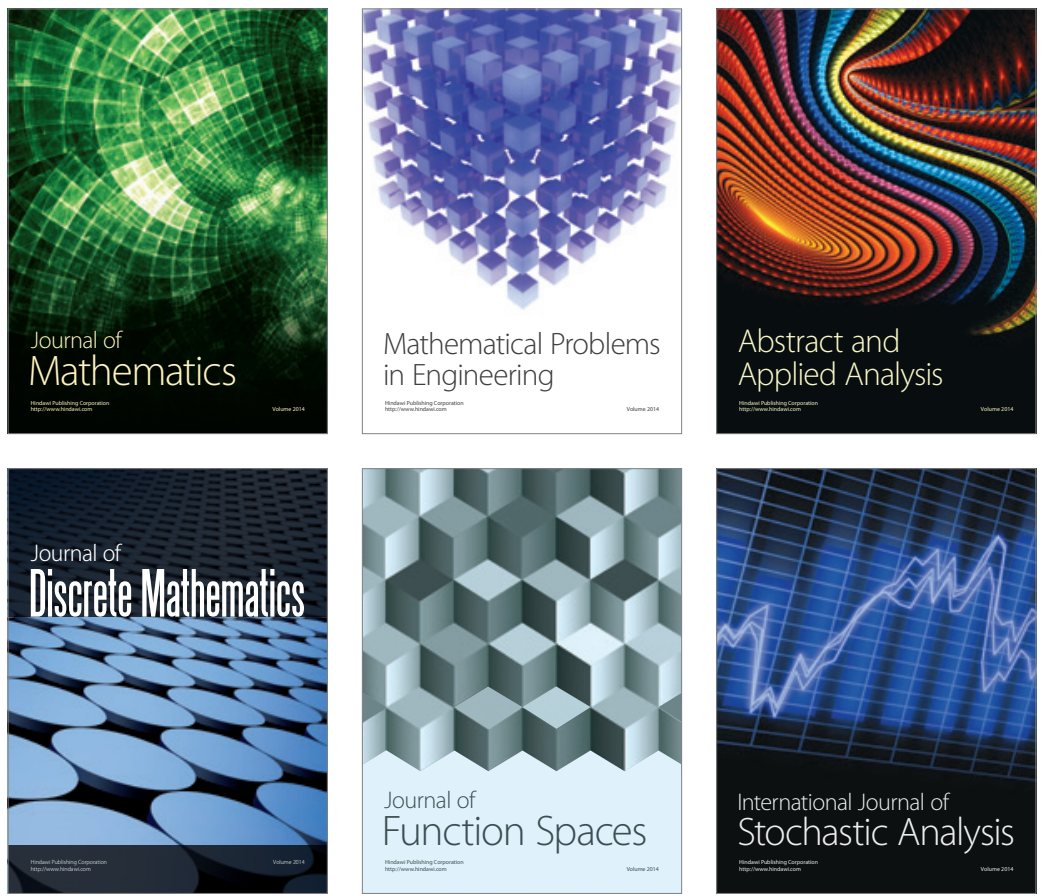

Journal of

Function Spaces

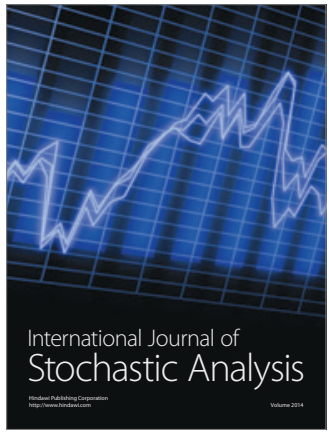

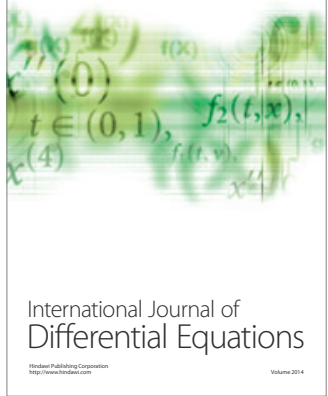
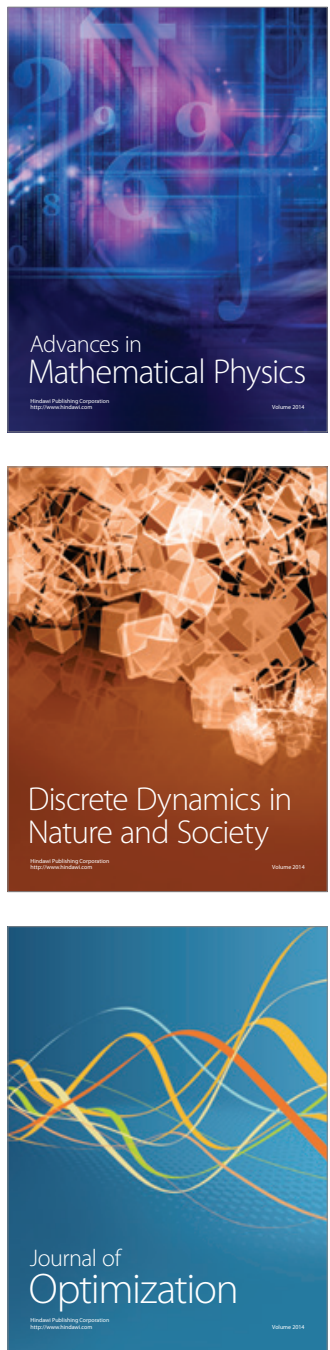